\title{
Razones de profesores de inglés para publicar en una revista y las lógicas del razonamiento sociocientífico
}

Motives of English Teachers to Publish in a Journal and the Logic of Socioscientific Reasoning

Razões para os professores de inglês publicarem em uma revista e a lógica do raciocínio sociocientífico

\section{Melba Libia Cárdenas* iD https://orcid.org/0000-0003-0028-2506}




\section{Resumen}

La publicación de artículos en revistas científicas o académicas ha alcanzado gran preponderancia en las comunidades académicas y en los indicadores de calidad principalmente de las instituciones universitarias. Presentamos las razones que llevaron a un grupo de docentes de inglés a enviar sus trabajos para publicar en una revista editada en Colombia. La información se recopiló mediante un cuestionario utilizado en un estudio de caso exploratorio. Analizamos los resultados desde la perspectiva de las lógicas del razonamiento científico-social implícitas en las opiniones de los autores y señalamos algunas implicaciones para la circulación de distintas voces en revistas editadas en países periféricos.

\section{Palabras clave}

lógica comunicativa; lógica crítica; lógica instrumental; publicaciones seriadas; escritores

\section{Keywords}

communicative logic; critical

logic; instrumental logic; periodical publications; writers

\begin{abstract}
The publication of articles in academic or scientific journals is highly relevant for academic communities and for attaining quality of institutions of higher education. We present the reasons that encouraged a group of teachers of English to submit their manuscripts for publication in a journal edited in Colombia. The data were gathered through a questionnaire applied in an exploratory case-study. We analyze the results from the perspective of the socioscientific reasoning implicit in the authors' opinions and highlight some implications that may have a bearing on the circulation of different voices in the journals being edited in periphery countries.
\end{abstract}

\begin{abstract}
Resumo
A publicação de artigos em revistas científicas ou acadêmicas se destaca nas comunidades acadêmicas e nos indicadores de qualidade, principalmente das instituições universitárias. Apresentamos as razões que levaram um grupo de professores de inglês a encaminhar seu trabalho para publicação em uma revista editada na Colômbia. As informações foram coletadas por meio de um questionário utilizado em um estudo de caso exploratório. Analisamos os resultados sob a perspectiva das lógicas de raciocínio científico-social implícitas nas opiniões dos autores e assinalamos algumas implicações para a circulação de diversas vozes em revistas publicadas em países periféricos.
\end{abstract}

\section{Palavras-chave}

lógica comunicativa; lógica crítica; lógica instrumental; publicações seriadas; escritores 


\section{Introducción}

La relevancia que tiene actualmente la divulgación de conocimientos a través de revistas científicas o académicas ha incidido en la financiación de universidades y centros de investigación, en los méritos otorgados tanto al profesorado como a las instituciones educativas, en las políticas y procesos editoriales y en el empeño de las publicaciones académicas o científicas por alcanzar su inclusión en sistemas de indexación y referenciación (SIR) o en reputadas bases bibliográficas. Este es un reto principalmente para las revistas editadas en países extramuros o periféricos como Colombia, donde se evidencian esfuerzos por consolidar sus revistas. Como muestra el último estudio sobre indicadores de ciencia y tecnología en Colombia (Lucio-Arias et al., 2015), de las 515 revistas clasificadas en el Índice Bibliográfico Nacional (IBN) Publindex, 208 son del área de ciencias sociales y 71 de humanidades. Del grupo de ciencias sociales, 31 revistas se centran en temas de educación y 17 del grupo de humanidades tratan sobre idiomas y literatura. Tres de este último grupo publican en inglés; una de ellas es Profile: Issues in Teachers' Professional Development (en adelante Profile, o la revista), editada por la Universidad Nacional de Colombia. Con todo, aún no conocemos el efecto de las más recientes políticas nacionales de evaluación de revistas (Colciencias, 2016a, 2016b) en los citados indicadores.

Dichas políticas establecen que la clasificación de las revistas depende principalmente de su presencia en los SIR y del impacto que los artículos puedan demostrar, con cifras concretas, es decir, con citaciones recibidas. A su vez, los debates en torno a los retos que enfrentan los docentes-investigadores para publicar apuntan al desconocimiento de las particularidades de las distintas disciplinas, como las ciencias sociales y humanas (Giménez-Toledo, 2014; Quintas-Froufe, 2016) y a la prioridad que se otorga en las políticas actuales a la necesidad de asegurar la visibilidad de la ciencia que se produce en Colombia en el ámbito internacional (Colciencias, 2016b). Por otra parte, se plantean también cuestionamientos respecto al efecto de las publicaciones en la carrera profesional docente (Cárdenas, 2014) e incluso se habla de la carrera de profesores universitarios por el puntímetro, como si la razón central para publicar fuera el deseo de obtener puntos que se revierten en incrementos salariales (Gómez-Morales, 2015). Esos debates también se han planteado, aunque de manera tangencial, en foros de editores y de profesores del área de lenguas. Sin embargo, no se conocen investigaciones que den cuenta de las diversas motivaciones que llevan a los autores a emprender el complejo proceso de presentar un manuscrito a una revista, para lograr su publicación. 
Realizamos un estudio de caso exploratorio (Simons, 2011; Yin, 2009) con el fin de conocer algunas experiencias de sus autores durante los procesos de evaluación de sus artículos, el significado atribuido a la meta alcanzada y las razones que los motivaron a presentar sus manuscritos para publicar en la revista. Este último aspecto constituye el eje del presente texto.

Los resultados permiten avanzar en el campo de la cognición del profesorado de lengua inglesa, a partir de las relaciones que se logran establecer con las tres lógicas del razonamiento sociocientífico, a saber: instrumental, comunicativa y crítica. Podemos establecer si la principal aspiración de los autores radica en la obtención de incentivos económicos o ascensos o si, por el contrario, existen otras razones que trasciendan el plano instrumental. Asimismo, se pueden vislumbrar implicaciones para fortalecer los procesos editoriales de las revistas, para incentivar la participación de diversas poblaciones de autores en ellas y fomentar la cultura de la publicación y la investigación en el campo de la escritura académica, entre otros.

\section{Marco conceptual}

Partimos de la definición de razón como principio de explicación de las realidades, como equivalente al fundamento, a los motivos que nos llevan a obrar (Ferrater Mora, 2009). Así, las respuestas de 67 autores de Profile nos acercan a sus experiencias y muestran enlaces con las tres lógicas del razonamiento sociocientífico: instrumental, comunicativa y crítica (Sabirón, 2007). Cada una de ellas se sustenta en teorías explicativas sobre un mismo fenómeno y nos retan a establecer relaciones con distintos escenarios. Advertimos que lejos de pretender realizar un tratado filosófico o epistemológico, examinaremos en qué consiste cada una de esas lógicas de razón y cómo se conectan con nuestro tema de estudio, sin establecer grados de supremacía.

Desde la perspectiva habermasiana, Sabirón (2007) establece una comparación con los condicionantes que tienen en nuestra vida las reglas del sistema al que pertenecemos (figura 1). Encontramos dos ejes que enmarcan la construcción del conocimiento científico-social: el sistema y el mundo de vida. El primero está constituido por las reglas del sistema al que pertenecemos. El segundo, también denominado comunicativo, se refiere a la persona, a su fuerza individual, la reconoce como parte de colectivos, organizaciones, instituciones y sistemas. Si pensamos en la relación entre esos dos planos, podemos imaginar "la tensión entre ambas fuerzas capaces de contextualizar el sentido y significado que podemos conferir a nuestras distintas acciones" (Sabirón, 2007, p. 37). La tríada se completa con la tensión, contradicciones y conflictos entre los dos 
primeros ejes, desde cuyas perspectivas se consideran las lógicas de razonamiento científico-social. Como explica el mismo autor, nos hallamos frente a "modos de razonamiento instrumental desde la perspectiva del sistema, comunicativa para el mundo de vida, e ideológica en la tensión a la que cada individuo, grupo, organización e institución responden" (p. 38). Así, tres esquemas dominantes rigen la razón: eficientista en la lógica instrumental; interpretativo o hermenéutico en la comunicativa; y dialéctico y crítico en la ideológica.

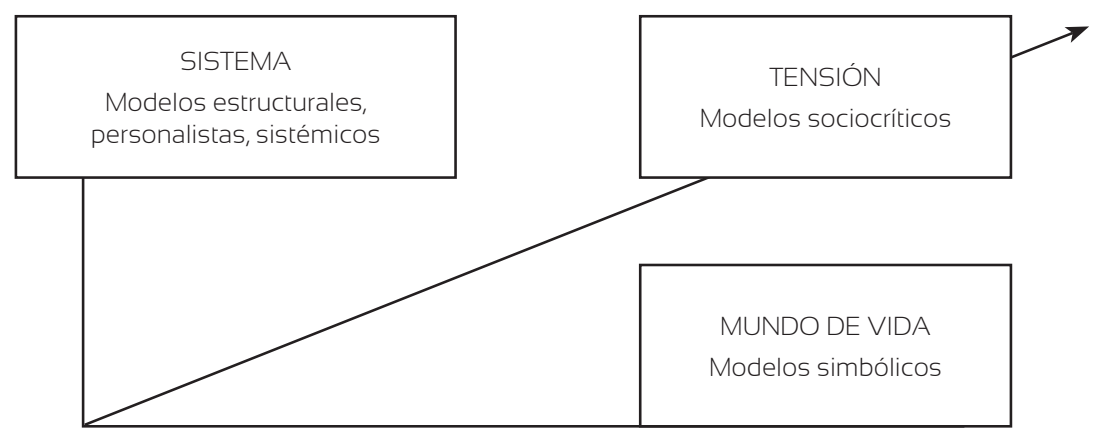

Figura 1

El sentido de la teorización en el ámbito científico-social

Fuente: Sabirón (2007, p. 40).

Los tres ejes se relacionan con los tres paradigmas o tradiciones intelectuales que enmarcan las prácticas investigativas en el campo de la educación: ciencias empírico-analíticas, ciencias simbólicas y ciencias críticas (Popkewitz, 1988). Los tres paradigmas están presentes en los artículos que se han publicado en la revista. Las primeras tradiciones intelectuales son de tipo positivista y propenden por la búsqueda de regularidades. Este es el caso de artículos referidos a intervenciones focalizadas en el aula, con miras a la mejora. En las segundas se examina la interacción entre los individuos para llegar a determinadas metas -como ocurre en artículos acerca del proceder de los actores educativos- y para ello se adopta un enfoque hermenéutico-interpretativo (Briones, 2002). En las ciencias críticas se estudian las relaciones sociales, el papel de los individuos y los factores que subyacen en el desarrollo histórico de un fenómeno -esto está presente principalmente en los artículos de reflexión y de revisión de temas. Además, en algunos escritos encontramos acercamientos tanto a las ciencias simbólicas como a las críticas, principalmente en los referidos a políticas educativas, relaciones de poder o indagaciones sobre la construcción de sentido en el marco de la interacción con determinados contenidos o en contextos particulares. 
En relación con la situación científica en las ciencias sociales, Sabirón (2007) identifica dos posiciones excluyentes: una focalizada en la explicación generalizable al sistema y otra en la comprensión en profundidad del mundo de vida. Entre ellas dos ubicamos un tercer eje emergente, que insta a superar las dicotomías, a considerar la diversidad de corrientes de pensamiento, la complejidad fenoménica. Esta tríada, representada por la tensión, contradicciones y conflictos entre los ejes sistema y mundo de vida, y el reconocimiento de una perspectiva sociocrítica, constituye un marco de referencia desde el que se consideran las lógicas de razonamiento científico. "La motivación para uno de los modos de razón es coherente con el eje sistémico o fenomenológico, enunciándose los tres tipos bajo un imperativo que rige la razón: eficientista en el primer caso, interpretativo para el segundo y crítico en el tercero" (Sabirón, 2007, p. 38). Esto nos reta a trascender el estudio de los fenómenos desde la sola perspectiva causa/ efecto, para acercarnos a la fenomenología, entendida como manera de ver el mundo, describiendo lo que se presenta en él, haciendo referencia a objetos concretos y a una trama de sentidos (Briones, 2002). Así podremos ahondar en la explicación, la comprensión descriptiva, interpretativa y hermenéutica. En el caso que nos ocupa, el análisis de los motivos que llevaron a 67 docentes de inglés a presentar sus manuscritos a Profile nos ubica en los tres planos: en las lógicas de razón instrumental, comunicativa y crítica.

\section{Método}

Enviamos un cuestionario corto por correo electrónico a todos los 312 autores que habían publicado en la revista entre 2000 y 2012, con el fin de identificar los motivos que los Ilevaron a enviar sus artículos a la revista, sus experiencias en los procesos de evaluación de sus manuscritos, las dificultades y unas primeras reflexiones sobre el significado que tuvo para ellos el que finalmente se publicaran. El cuestionario fue uno de los instrumentos empleados en un estudio de caso exploratorio (Simons, 2011; Yin, 2009) que forma parte del estudio de línea base de la tesis doctoral en curso y cuya temática gira alrededor de dicha revista. El cuestionario fue de tipo transversal seccional, de prevalencia o cross-sectional puesto que los datos se recopilaron en un solo momento temporal, para luego realizar una descripción de los asuntos en cuestión (Creswell, 2009).

Se recibieron respuestas de 67 autores. Como ya se indicó, nos centramos en el análisis de los resultados del primer ítem del cuestionario: "¿Qué lo hizo decidirse a presentar su(s) artículo(s) para publicación en la revista?". 


\section{Contexto de la investigación y participantes}

La revista se creó en el año 2000, con una periodicidad anual y en 2008 cambió a semestral. Su propósito se evidencia en las tres secciones que la caracterizan: temas de docentes investigadores, de nuevos docentes investigadores y basados en reflexiones e innovaciones. En cuanto a su visibilidad, la publicación está registrada en diversos sistemas de referenciación e indexación internacionales, así como en el Índice Bibliográfico Nacional Publindex.

Los 67 encuestados son profesores de inglés y sus artículos versan sobre la enseñanza del inglés en diferentes niveles educativos, la formación del profesorado y políticas lingüísticas. La mayoría (70 \%) labora en universidades; el 20 \%, en educación primaria o secundaria; el 7 \% culminó recientemente su licenciatura en lenguas y unos pocos (3\%) trabajan en institutos de idiomas o de forma independiente. En cuanto a su procedencia, son de Colombia (55), Estados Unidos (3), Gran Bretaña (1), India (2), Italia (1), México (3), Turquía (1) y Ucrania (1).

\section{Análisis de datos}

Realizamos codificación abierta (Strauss y Corbin, 1990). Además, y puesto que se tuvo un papel de observadora-participante -la investigadora es la misma editora de la revista-, se buscó la eliminación o control de prejuicios mediante la clarificación de las suposiciones, visiones del mundo y orientaciones teóricas a la luz del estudio. Asimismo empleamos procesos de restitución al campo o confrontación de pares. Para ello compartimos el primer manuscrito de este artículo con diez participantes y obtuvimos retroalimentación de seis de ellos.

\section{Resultados}

La tabla 1 muestra las categorías y subcategorías y la frecuencia de respuestas en los temas centrales. Al respecto de los motivos para publicar en la revista, advertimos que son aquellos que los encuestados aseveraron tener $y$, como en toda investigación que incluya opiniones, las respuestas pueden estar sesgadas o influidas por el contexto social o laboral. 
Tabla 1

Razones de los autores para publicar

\begin{tabular}{lll}
\hline \multicolumn{1}{c}{ Categorías } & $\%$ & \multicolumn{1}{c}{ Subcategorías } \\
\hline $\begin{array}{l}\text { Deseo de contribuir a la } \\
\text { comunidad académica }\end{array}$ & 45 & $\begin{array}{l}\text { Colaboración con pares } \\
\text { Aporte de utilidad } \\
\text { Interés local }\end{array}$ \\
Visibilidad de la publicación & 31 & $\begin{array}{l}\text { Prestigio } \\
\text { Temática } \\
\text { Inclusión de voces } \\
\text { Rigor }\end{array}$ \\
Logro de retos & & $\begin{array}{l}\text { Ciclo investigativo } \\
\text { Estatus } \\
\text { Acompañamiento que se } \\
\text { brinda }\end{array}$ \\
\hline
\end{tabular}

Fuente: elaboración propia.

El compromiso de aportar a la comunidad docente, con trabajos pertinentes es el principal factor que incita a los docentes a enviar sus contribuciones. Motiva también el nivel de circulación de la revista y, en menor proporción, el respaldo que les brinda para asumir el reto de publicar. Por último, unos pocos profesores expresan que actúan movidos porque desean alcanzar una meta que a su vez les garantiza algún beneficio práctico.

El análisis de los ejes semánticos en los que se agrupan las razones expresadas por los participantes mostró que no hay fronteras completamente delimitadas entre ellas. A la luz del modelo de las lógicas del razonamiento científico-social (Sabirón, 2007), algunas razones pueden ubicarse en uno de los tres planos de razón; otras hacen explícito el nexo con motivos puntuales inmersos en otra lógica o con el sentido global que emana de otro plano. Este es el caso de respuestas referidas a la temática, la inclusión de diversas voces, la voluntad de aportar a la comunidad académica y el logro de retos (figura 2).

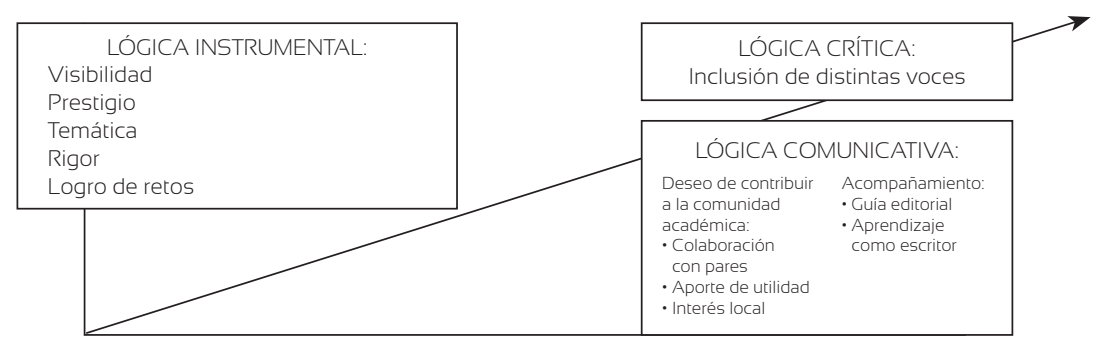

Figura 2

Relación entre las razones para publicar y las lógicas instrumental, comunicativa y crítica Fuente: elaboración propia. 
Asociamos las razones expresadas por los autores con los planos instrumental, comunicativo y, en menor proporción, de la ciencia inclusa o sociocrítica. En el primero encontramos el reconocimiento del prestigio y la seriedad de la revista. En el segundo, hallamos el compromiso por comunicarse con la comunidad académica. Allí subyacen motivos personales y profesionales. En cuanto a la lógica crítica, se perciben motivos relacionados con la transformación de la sociedad del conocimiento, compromisos éticos y profesionales del docente/investigador; el interés por estudiar la complejidad de la labor educativa, formar comunidad y lograr cambios en la enseñanza del inglés. A continuación examinamos cada plano de razón e ilustramos cada uno con algunos extractos de las respuestas de los participantes, traducidos al castellano e identificados con seudónimos.

\section{Lógica instrumental}

La visibilidad es el principal motivo por el cual los autores seleccionan la revista para enviar sus manuscritos. Esta categoría está relacionada asimismo con la calidad y el rigor. El prestigio se entiende como el reconocimiento que tiene la revista principalmente en Colombia y la visibilidad internacional que ha alcanzado, sobre todo, en países latinoamericanos. A su vez, la rigurosidad que perciben los autores en el trabajo que realiza la revista está asociada al reconocimiento de los méritos académicos de los miembros de sus comités y de los pares evaluadores que la apoyan.

En segunda instancia, hallamos la temática, vital en la toma de decisiones. Si bien esto puede parecer obvio, un alto número de autores estima que deseaban publicar en la revista porque los contenidos son pertinentes para la comunidad académica. En especial, destacan el hecho de que traten la formación del profesorado y la enseñanza del idioma desde las perspectivas de la innovación y la investigación, debidamente fundamentadas.

Pienso que [la revista] se centra en temas que necesitan debatir los profesores de lenguas extranjeras y va más allá de la presentación de juegos o actividades sin sustento teórico. (Celia)

Celia resalta que se propenda por superar los imaginarios que circulan a menudo sobre el docente de idiomas: alguien que debe preocuparse principalmente por propiciar la diversión en el aprendizaje de un código lingüístico. Su percepción concuerda con Cortés, Cárdenas y Nieto (2013), quienes equiparan al docente de idiomas con la figura del mago: debe ser a la vez un científico y un artista; debe poseer no solamente saberes teóricos y prácticos, sino la habilidad para solucionar problemas y para crear.

Por otra parte, el prestigio de la revista, la probabilidad de llegar a una amplia audiencia y el deseo de alcanzar méritos profesionales son una constante en las opiniones de un pequeño grupo de autores, mayoritariamente 
colombianos. Pareciera que en algunos de ellos hay un vínculo causa/efecto entre las políticas establecidas por Publindex y las razones que los llevan a seleccionar la revista. Así lo expresa un docente universitario:

Es una de las revistas mejor clasificadas en Colombia. Esto significa que lo que se publica tendrá un importante número de lectores. Así que tu contribución puede tener un impacto más amplio. Adicionalmente, no podemos negar que publicar en una revista indexada te da más puntos en tu carrera académica. (Juan)

Observamos que la obtención de méritos en la carrera profesional se traduce en aumento salarial. A su vez, se reconocen otras ganancias que motivan a asumir el reto:

Quería tener mi primer trabajo investigativo publicado, como parte de mi desarrollo y objetivos profesionales. (Dora)

Fue un reto personal y profesional escribir un artículo que cumpliera con todos los estándares de contenido y forma que exige la revista. (Francy)

Se puede obtener reconocimiento académico. (Jaime)

El incremento en el interés de los profesores por publicar en algunas de revistas está asociado a los sistemas de incremento salarial de los docentes universitarios y la acreditación institucional. Para esta última se tienen en cuenta, entre otros, indicadores relacionados con las publicaciones de sus docentes. Todo esto está asociado a la relevancia que hoy poseen la evaluación y la garantía de la calidad en la educación (Díaz Palacios, 2015). Si bien se observa una tendencia competitiva en los ámbitos académico e investigador de contextos como el europeo y el norteamericano, la tendencia permea a otras regiones. De hecho, países periféricos como Colombia propenden por modelos eficientistas que instan a sus académicos a una mayor producción, sobre la base de la competitividad (Delgado, 2011; Lillis y Curry, 2010). Así, desde la lógica instrumental se establecen criterios de control de calidad tanto para el sistema educativo como para el control interno de lo que allí se produce. Esto se asocia con los fundamentos que explican la presencia de la razón instrumental en los modelos estructurales y funcionalistas de los sistemas educativos o de los que rigen las políticas para publicar en revistas de regiones hegemónicas y la clasificación de las publicaciones seriadas. En la mayoría de los casos priman el interés por estimar los impactos cuantitativos de la inversión en educación, la circulación de trabajos de autores de determinadas élites y el funcionamiento supeditado a modelos de intervención, desarrollo y calidad. 


\section{Lógica comunicativa}

Recordemos que la lógica del mundo de vida o comunicativa se refiere a la persona, a su fuerza particular, cuyo accionar se halla también relacionado con los colectivos a los que pertenece o propios de su entorno. La mayoría de los encuestados ejerce su derecho a divulgar su trabajo en un medio determinado, pues consideran que es posible llegar a otros docentes a través de la publicación de experiencias, reflexiones o investigaciones referidas a su contexto educativo. De aquí se desprende que las razones de los autores no son solo prácticas o de racionalidad técnica, hecho que nos ubica en la razón subjetiva, en cuanto a comunicativa, frente a la razón funcional. En este sentido, nos ubicamos en una dimensión social. Según Reyes (2009, p. 2623), para Habermas la racionalidad de la acción social debería entenderse

[...] desde una teoría de la comunicación en la que no se trata, ante todo, de actos comunicativos, sino de la coordinación de las acciones extralingüísticas teleológicas por la vía de una racionalidad que no es precisamente medio-fin, sino de la comprensión a través de actos lingüísticos de comunicación.

La tabla 1 muestra que en el $45 \%$ de los autores encuestados prima un interés por compartir los resultados del trabajo realizado con docentes que tienen intereses similares y con la comunidad académica en general. Sandy expresa su decisión

[...] de compartir mis hallazgos e investigación con personas cuyos intereses son similares a los míos, ya que podrían estar más dispuestos a apreciar y a darle un buen uso a dicha información.

En contraste, si bien solo dos autores reconocen un motivo instrumental -la obligatoriedad de la escritura del artículo de su autoría, como requisito de un curso de desarrollo profesional para docentes de colegios de primaria y secundaria que realizaron-, admiten la importancia de aportar a otros docentes. Notamos que, en general, los mueve el convencimiento de que los contenidos de la revista son útiles: "Pensé que tenía algo relevante y posiblemente útil que decir" (Isabel). A su vez, a cinco de ellos los motiva la certeza de que como docentes-investigadores tienen algo que aportar a la construcción de conocimiento local. Así lo ilustran dos autoras noveles, cuyos artículos se constituyeron en su primera publicación, producto también de un acercamiento inicial a la investigación educativa:

Algunos profesores investigadores lo han leído como un punto de referencia para llevar a cabo sus trabajos de investigación. (Yamile) 
Nuestro artículo está basado en una investigación que llevamos a cabo como trabajo de grado para obtener el título de licenciatura en la Universidad. Como el objetivo final al hacer investigación es construir conocimiento y compartir experiencias, encontramos que merece la pena compartir nuestros hallazgos y experiencias con quienes están involucrados en el proceso de enseñanza/aprendizaje de una lengua extranjera. (Sally)

Se percibe que si socializan los resultados de la investigación se contribuye a la constitución de comunidad y se estable una interacción con pares. A su vez, una docente de educación secundaria resalta:

El hecho de compartir con la comunidad académica lo que he aprendido desde la experiencia académica o el proceso investigativo que he llevado a cabo. [...] Debemos recordar que la acción de escribir es mucho más significativa cuando se tiene a alguien que pueda escuchar, apoyar o refutar tus ideas. (Isabela)

Los anteriores testimonios se hallan en consonancia con los postulados de Habermas (2001), quien argumenta que el conocimiento es producto de nuestras preocupaciones íntimamente relacionadas con la cotidianidad. En el caso de las profesoras, el conocimiento proviene de su labor docente, del eje de un proyecto que se resume en un artículo. Además, las expectativas de las autoras por establecer un diálogo con sus lectores y contribuir a una comunidad académica muestran la dimensión social que, según Habermas, comprende las relaciones entre los seres humanos, centrada en la cultura y en las normas sociales. Como explican Alvarado y García (2008):

Para Habermas (1986) el conocimiento nunca es producto de individuos o grupos humanos con preocupaciones alejadas de la cotidianidad; por el contrario, se constituye siempre en base a intereses que han ido desarrollándose a partir de las necesidades naturales de la especie humana y que han sido configurados por las necesidades históricas y sociales. (p. 191).

Las opiniones de las mencionadas participantes no se circunscriben a la mejora. Por el contrario, reflejan la lógica comunicativa en tanto sus apreciaciones respecto a la razón por la cual decidieron publicar sus artículos radican en un intento por tratar de clarificar asuntos o realidades determinados y aportar a otros. Esto es posible gracias al hecho de involucrarse como participantes y observadores en un contexto que les permite captar registros, percepciones, actitudes, valoraciones e interpretaciones de los actores y eventos involucrados en los trabajos que reportan en sus escritos. Posteriormente, la interacción con otros se amplía durante el proceso editorial, especialmente con los pares evaluadores y editores. Se trata entonces de la interpretación simbólica de los protagonistas. A este tenor, recordemos 
que a comienzos del siglo xx John Dewey argumentaba que todos somos conocedores, reflexionamos acerca de las experiencias, confrontamos lo desconocido, hacemos sentido de él y tomamos acciones. Así, los profesores como conocedores y productores legítimos de conocimiento, se comprometen con su desarrollo profesional a lo largo de la vida y contribuyen al fortalecimiento de comunidades de docentes/escritores.

Sabemos que la acción dialógica se amplía al emprender la publicación de nuestros trabajos. Si bien es una forma de compartir ideas y probablemente hacer alguna contribución al conocimiento existente, publicar presupone un diálogo con interlocutores que no necesariamente conocemos (Benson, 2000). En nuestro caso, el diálogo se establece en primera instancia con los equipos editoriales, donde cumplen un papel fundamental los pares evaluadores como acompañantes del proceso de publicación de un texto. Así, un pequeño porcentaje de los encuestados (9 \%) alude que el acompañamiento y la guía editorial son razones primordiales para enviar sus trabajos a la revista. En ellos encuentran también oportunidades para lograr aprendizajes como escritores y guía para que los investigadores en formación hagan su labor: "Creo que he aprendido a ser una mejor escritora y editora [de los trabajos de mis estudiantes-docentes de un programa de licenciatura]" (Mercy).

Vemos la conciencia de los formadores de docentes como escritores y maestros de otros docentes y reconocemos también la función que les corresponde a las publicaciones académicas en este mismo sentido por cuanto se trata no solo de brindar seguridad a los escritores, sino de actuar en consonancia con la necesidad de acompañar a las comunidades académicas en su empeño por ganar voz. A la luz del interaccionismo simbólico (Briones, 2002; Habermas, 2001), el interés de autores y de la revista por dar cuenta del sujeto y contribuir a la comunidad educativa nos muestra una concepción de lo social como el marco de la interacción simbólica de individuos y de la comunicación como el proceso social por excelencia que puede aportar a la constitución o avance de los grupos (las comunidades académicas) y los individuos (los profesores/escritores).

Finalmente, y puesto que la producción y circulación de los escritos de los docentes de inglés no tiene una amplia trayectoria en países periféricos, es preciso subrayar que no se trata solamente de producir un buen manuscrito, seguir las directrices de la publicación o asegurarse de cumplir determinados estándares. En otras palabras, y retomando a Habermas (1988, 2001), no se trata de regirnos por la imposición de la racionalidad del sistema al mundo de vida, pues a los profesores/escritores no los motiva únicamente su éxito individual, sino la armonización de sus acciones sobre la base de definiciones comunes -en nuestro caso, el interés conjunto de una comunidad profesional-. 


\section{Lógica crítica}

Hemos señalado que en las razones de los autores asociadas a la lógica instrumental prevalece una relación causa-efecto y en las relacionadas con la lógica comunicativa predominan la posibilidad de llegar a "una comprensión descriptiva, interpretativa y hermenéutica" (Sabirón, 2007, p. 38). También anotamos que hay conexiones entre algunas razones expresadas por los autores que justamente nos llevan a pensar en las tensiones que se generan entre los campos que abarcan las dos primeras lógicas. Así, la tercera lógica o razón crítica nos insta a superar las dicotomías y a considerar la complejidad, entendida como "la unión entre la unidad y la multiplicidad" (Morin, 1999, p. 16), que se espera permita un modo de conocimiento capaz de aprehender los objetos en sus propios ámbitos, sus complejidades y sus conjuntos. Ese tercer eje, sociocrítico, nos muestra "la naturaleza ideológica que impregna el sentido de la acciones sociales y educativas bajo un esquema dinámico, interactivo y dialéctico" (Sabirón, 2007, p. 38).

Los testimonios de algunos encuestados reflejan que hay una pluralidad de individuos en la comunidad académica, cuya diversidad cultural debería ser acogida en las revistas científicas. En este sentido, reconocen la trascendencia de un espacio para publicar, donde se escuchen voces de profesores de distintos niveles. Algunas autoras opinan:

Me gusta el hecho de que la revista se dedica a hacer que se escuchen las voces de los maestros y yo quería ser parte de esa comunidad. (Hilda)

Está dirigida a la investigación acción de profesionales de base y docentes en formación, quienes constituyen una comunidad muy olvidada en nuestro campo. (Isabel)

No solo los profesores investigadores pueden publicar allí sino también docentes investigadores noveles. (Bianca)

Al respecto cabe señalar que la tendencia del profesor investigador concede importancia a los procesos sistemáticos e intencionales de cuestionamiento de los docentes, que no se originan únicamente en la teoría o en la práctica, sino en la reflexión crítica que genera la intersección entre ellas, independientemente de que se trate de asuntos puntuales, sencillos o de mayor envergadura (Cochran-Smith y Lytle, 1993; Golombek y Johnson, 2017). Se reconocen también las teorías que poseen los docentes y sus interpretaciones personales. Así se posibilita que tanto las comunidades universitarias como escolares tengan acceso a la experticia de los docentes y desarrollen perspectivas singulares acerca de procesos educativos que no pretenden generalizar sino, más bien, empoderarlos (Kincheloe, 2003). 
El empoderamiento de los docentes presupone que tengan un papel más activo en las decisiones de las instituciones educativas, con lo cual se busca darles un mayor control sobre su trabajo, una voz en su profesión (Zeichner, 2010). En el contexto colombiano, donde laboran la mayoría de los autores encuestados, se ha encontrado que la actividad investigativa potencia la labor de los docentes de inglés, permite la desmitificación del papel del investigador, el cuestionamiento constante para la toma de decisiones y el cambio, así como la valoración de la investigación como una vía para el desarrollo profesional (Cárdenas, 2004, 2014; Cárdenas y Nieto, 2010; Vergara, Hernández y Cárdenas, 2009). Esta concepción crítica reconoce la experiencia y creatividad del profesor para afrontar y desarrollar asuntos identificados en su contexto laboral, en el que la reflexión y el cuestionamiento de creencias, valores y prácticas aportan al crecimiento personal y profesional (Cárdenas, González y Álvarez, 2010). A este propósito, Myriam asevera:

Estamos acostumbrados a hacer algunas cosas en nuestra labor diaria y a veces pensamos que no vale la pena compartirlas de una manera formal. Sin embargo, algo que he aprendido a lo largo de mi experiencia con Profile, no solo como autora, sino como docente-estudiante [de un programa de desarrollo profesional para profesores de secundaria] y posteriormente como formadora de docentes [en ese programa] es que el nuevo conocimiento no crece en mentes brillantes, sino en personas reales, informadas, con perspectivas y reflexivas.

Myriam alude al hecho de que los individuos no tienen por qué conocerlo todo, pero pueden reportar el conocimiento que poseen, informados por los contextos que les son propios. Además, relativiza la superioridad de los que se consideran poseedores del conocimiento y sitúa al docente en el ámbito de la investigación en el aula, como planificador de decisiones, reflexivo y con capacidad de vincular su área de enseñanza con la formación, actualización y conciencia histórica de la realidad en donde emerge. Se reconocen así oportunidades para indagar, mediante el cuestionamiento narrativo (narrative inquiry) y contextualizado (Golombek y Johnson, 2017), las creencias, conocimientos y experiencias previas de los docentes. Por otra parte, y desde las perspectivas socioconstructivista (Sabirón, 2010) y sociocrítica (Alvarado y García, 2008), Myriam refleja la confianza en la capacidad reflexiva del profesorado, la búsqueda de su emancipación y el convencimiento de que es posible investigar y divulgar el conocimiento. En este sentido, resaltamos la vigencia de Cataño (1992), quien expresa que "el ejercicio cotidiano del profesor universitario es de gran ayuda para sus publicaciones impresas" (p. 16). 
Sin embargo, no siempre se publican los resultados de sus investigaciones y poco conocemos acerca de las circunstancias en las que las realizan o sobre las vivencias de quienes logran publicarlas. Además, aunque dentro de contextos locales se ha venido cediendo vOz a los profesores, pareciera que las comunidades dominantes en la enseñanza del inglés no les prestan importancia. Tampoco se sabe con certeza si los resultados de las indagaciones de los profesores de base tienen acogida en esas comunidades por cuanto se asocian primordialmente con los asuntos prácticos de la docencia (Cárdenas y Rainey, 2017). De ahí la necesidad de la formación de una identidad individual de carácter crítico, del reconocimiento de la singularidad de los profesores de lenguas extranjeras (Cortés, Cárdenas y Nieto, 2013) y el fortalecimiento de la cultura de la escritura tanto en aquellas comunidades ya constituidas, sin una tradición en la circulación del conocimiento por vía de las publicaciones, como en los programas de formación del profesorado. Finalmente, precisamos avanzar en la investigación referida a la línea de escritura académica pues, como advierten Níkleva y Cortina Pérez (2014, p. 282), "Ios metaanálisis sobre la producción científica son escasos y no actualizados".

\section{Conclusiones e implicaciones}

Nos hemos aproximado a las lógicas de razonamiento sociocientífico para comprender las razones que motivaron a profesores de inglés a publicar sus artículos en Profile y a posibles implicaciones para mantener su presencia en la revista. Entre los motivos prima la lógica comunicativa, por cuanto interesa el vínculo con la comunidad académica. Asimismo, y en relación con el plano instrumental, los participantes reconocen el efecto de una publicación en una revista reputada: la satisfacción personal, el reconocimiento de sus pares académicos y, en unos pocos casos, los incentivos que se pueden obtener para la carrera profesional, representados en el escalafón docente o en salario. A su vez, y aunque en menor proporción, se encuentran argumentos asociados a la lógica crítica, en tanto reflejan el compromiso de los autores como agentes comprometidos con el cambio educativo, la generación de conocimiento y la investigación en su campo profesional.

Los resultados pueden, por un lado, brindar elementos de referencia a los equipos editoriales de las revistas del área y, por otro, permitir a los profesores de inglés conocer acerca de las vivencias de docentes/investigadores como escritores. Además, señalan aspectos relevantes para la formación del profesorado.

En cuanto al trabajo editorial, el interés por liderar revistas científicas plantea nuevos retos para las comunidades académicas, cuyos productos deben competir con empresas editoriales con amplia experiencia en 
los campos de la edición y del mercadeo. Las universidades, que en su mayoría editan revistas como una actividad agregada a aquellas que le son propias, motivan a publicar conforme a los índices de calidad de sus especialidades y las políticas gubernamentales o de los SIR, pero también tienen un compromiso en el fomento de la cultura de la escritura y la publicación Desde ese lugar privilegiado, pero también complejo, probablemente se podrá aportar a estudios sobre la escritura académica, la cognición del profesorado, la concepción y desarrollo de programas de formación permanente, y los procesos editoriales que median la edición de revistas académicas, entre otros.

Los hallazgos antes presentados pueden permitirnos ampliar nuestros conocimientos acerca de los imaginarios de los docentes de inglés como escritores y autores de artículos. Esto puede llevar a los equipos editoriales de las revistas a una comunicación más fluida con sus autores, a aproximarnos a ellos desde ópticas que superen los procesos propios del flujo editorial, de tal suerte que podamos comprender las lógicas que subyacen la presentación de sus manuscritos, sus planteamientos y la interacción con los actores de la publicación (editores, pares evaluadores, revisores). Asimismo, es posible que los mismos docentes interesados en divulgar sus trabajos en las revistas comprendan los razonamientos de autores que, como ellos, buscan hacer visible su trabajo en revistas académicas.

Por otra parte, puesto que se busca motivar a los profesores de inglés a publicar, es preciso examinar qué tan familiarizados están con el campo de la escritura académica y con los procesos que acarrean la presentación, evaluación y publicación de un manuscrito. En este sentido, esperamos que nuestros hallazgos aporten a las rutas de formación inicial y permanente de los docentes pues, como se sabe, las competencias escriturales se desarrollan en diversos escenarios: al leer ciencia que se publica, al escribir y debatir en torno a temas propios de la profesión, entre otros.

Los resultados del presente estudio nos acercan a los imaginarios de un grupo de docentes y a sus vivencias como autores. En sus testimonios pudimos encontrar que, aunque posean el conocimiento específico de las temáticas que abordan en sus escritos, tienen también expectativas respecto al apoyo que pueden recibir para alcanzar sus propósitos. Requerimos entonces un debido acompañamiento durante el proceso editorial, con lo cual puede predecirse algún impacto en las comunidades académicas. De aquí se desprende que sería no solo interesante sino pertinente indagar luego acerca de la naturaleza de la retroalimentación que reciben los autores; cómo la perciben los autores; qué tipo de interacción se ha dado entre autores y evaluadores; la labor de mediación de la editora; y el efecto de estos procesos en el producto final (el artículo). 
Para finalizar, subrayamos que la comprensión de un fenómeno no consiste en entender al otro, sino en entenderse con otro sobre un texto (las respuestas brindadas por los docentes-autores en un cuestionario), teniendo presentes las circunstancias sociales e históricas en las que se circunscribe el asunto que nos interesa. Esta fusión de horizontes permite definir un horizonte más amplio del fenómeno. Asimismo, reconocemos que la comprensión de las respuestas de los participantes es una aproximación que hemos proyectado sobre el objeto de estudio y no está libre de prejuicios. Estas apreciaciones pueden ser modificadas por los encuestados si, por ejemplo, indagamos por experiencias más recientes. Puesto que toda comprensión se realiza dentro de un ciclo hermenéutico, las inquietudes que sigan surgiendo como resultado del presente análisis nos conducirían a una nueva comprensión del fenómeno, a una ampliación o a una nueva restauración del sentido de las ideas expresadas por los sujetos. Reconocemos que nuestras interpretaciones constituyen un proceso inacabado, pues no existe una única forma de interpretación de un hecho, de un argumento.

\section{Agradecimientos}

A los autores que contestaron el cuestionario y a los que amablemente leyeron la primera versión de este texto, como parte del proceso de restitución al campo. Igualmente, un reconocimiento al doctor Fernando Sabirón (Universidad de Zaragoza, España), por sus aportes en asuntos epistemológicos.

\section{Referencias}

Alvarado, L. y García, M. (2008). Características más relevantes del paradigma sociocrítico: su aplicación en investigaciones de educación ambiental y de enseñanza de las ciencias realizadas en el Doctorado de Educación del Instituto Pedagógico de Caracas. Sapiens. Revista Universitaria de Investigación, 9(2), 187-202.

Benson, M.J. (2000). Writing an Academic Article: An Editor Writes... Forum English Teaching, 3(2), 33-35.

Briones, G. (2002). Epistemología de las ciencias sociales. Bogotá: Instituto Colombiano para el Fomento de la Educación Superior (ICFES).

Cárdenas, M. L. (2004). Las investigaciones de los docentes de inglés en un programa de formación permanente. Ikala, Revista de Lenguaje y Cultura, 9(15), 105-137. 
Cárdenas, M.L. (2014). Publishing and academic writing: Experiences of authors who have published in Profile. Profile: Issues in Teachers' Professional Development, 16(2), 11-20. DOI: https://doi. org/10.15446/profile.v16n2.46144

Cárdenas, M.L. y Nieto, M.C. (2010). El trabajo en red de docentes de inglés. Bogotá: Universidad Nacional de Colombia.

Cárdenas, M.L., González, A. y Álvarez, J.A. (2010). El desarrollo profesional de los docentes de inglés en ejercicio: algunas consideraciones conceptuales para Colombia. Folios, 31, 49-67.

Cárdenas, M.L. y Rainey, I. (2017). Publishing from the ELT periphery: The Profile journal experience in Colombia. En M.J. Curry y T. Lillis (eds.), Global academic publishing: Policies, perspectives, and pedagogies (pp. 151-165). Clevedon, Reino Unido: Multilingual Matters.

Cataño, G. (1992). De la publicación oral a la publicación impresa. Estrategias para desarrollar la producción intelectual en la universidad. Revista Colombiana de Educación, 24, http://dx.doi. org/10.17227/01203916.5205

Cochran-Smith, M. y Lytle, S.L. (1993). Inside outside. Teacher research and knowledge. Nueva York: Teachers College Press.

Colciencias. (2016a). Convocatoria No. 768 de 2016 para indexación de revistas científicas colombianas especializadas - Publindex. Bogotá. Recuperado de http://www.colciencias.gov.co/sites/default/ files/upload/convocatoria/terminosdereferencia-conv768-2016-firmados.pdf

Colciencias (mayo de 2016b). Documento No. 1601: política para mejorar la calidad de las publicaciones científicas nacionales. Versión para discusión. Bogotá.

Cortés, L., Cárdenas, M.L. y Nieto, M.C. (2013). Competencias del profesor de lenguas extranjeras: Creencias de la comunidad educativa. Bogotá: Universidad Nacional de Colombia.

Creswell, J. (2009). Research design. Qualitative, quantitative and mixed methods and approaches. 3a. ed. Londres: Sage.

Delgado, J.E. (2011). Journal publication in Chile, Colombia, and Venezuela: University responses to global, regional, and national pressures and trends (Ph.D.). University of Pittsburgh, ProQuest Dissertations and Theses. Recuperado de http://search.proquest.com/docview/908423407? accountid $1 / 411652(908423407)$

Díaz Palacios, J.A. (2015). Re-significación y re-conceptualización de la categoría calidad educativa: una nueva mirada, una nueva perspectiva. Revista Colombiana de Educación, 68, 173-194. Dol: http://dx.doi. org/10.17227/01203916.68rce173.194 
Ferrater M., J. (2009). Diccionario de filosofía. Q|Z. 2a. ed. Barcelona: Ariel Filosofía.

Giménez-Toledo, E. (2014). Imposturas en el ecosistema de la publicación científica. Revista de Investigación Educativa, 32(1), 13-23. Dol: http:// dx.doi.org/10.6018/ rie.32.1.190251

Golombek, P.R. y Johnson, K.E. (2017). Re-conceptualizing teachers' narrative inquiry as professional development. Profile: Issues in Teachers' Professional Development, 19(2), 15-28. Dol: https://doi. org/10.15446/profile.v19n2.65692

Gómez-Morales, Y.J. (2015). Usos y abusos de la bibliometría 1. Revista Colombiana de Antropología, 51(1), 291-307.

Habermas, J. (1988). Teoría de la acción comunicativa. Tomo II. Crítica de la razón funcionalista. Madrid: Taurus.

Habermas, J. (2001). Teoría de la acción comunicativa. Tomo I. 2a. ed. Madrid: Taurus.

Kincheloe, J.L. (2003). Teachers as researchers. Qualitative inquiry as a path to empowerment. Londres: Routledge Falmer.

Lillis, T. y Curry, M.J. (2010). Academic writing in a global context: The politics and practices of publishing in English. Nueva York: Routledge.

Lucio-Arias D. et al. (2015). Indicadores de ciencia y tecnología, Colombia 2014. Bogotá: Observatorio Colombiano de Ciencia y Tecnología. Recuperado de http://ocyt.org.co/proyectos-y-productos/indicadores-de-ciencia-y-tecnologia-colombia-2014/

Morin, E. (1999). Los sietes saberes necesarios para la educación del futurO. París: UNESCO.

Níkleva, D.G. y Cortina Pérez, B. (2014). La producción científica actual en Didáctica de la Lengua y la Literatura en revistas españolas. Porta Linguarum: Revista Internacional de Didáctica de las Lenguas Extranjeras, 21, 281-299.

Popkewitz, T.S. (1988). Paradigma e ideología en investigación educativa: las funciones sociales del intelectual. Madrid: Mondadori.

Quintas-Froufe, N. (2016). Indicadores de calidad de las publicaciones científicas en el área de Ciencias Sociales en España: un análisis comparativo entre agencias evaluadoras. Revista de Investigación Educativa, 34(1), 259-272. Dol: http://dx.doi.org/10.6018/rie.34.1.210191

Reyes, R. (dir.) (2009). Diccionario crítico de ciencias sociales. Terminología científico-social. P-Z. Vol. 4. Madrid: Plaza y Valdés.

Sabirón, F. (2007). Métodos de investigación etnográfica en Ciencias Sociales. Zaragoza: Mira Editores. 
Sabirón, F. (2010). Sobre el cuestionamiento postmodernista en investigación etnográfica: galgos o podencos, son unos conejos. Ethnography and Education European Review / Revue Européenne d'Ethnographie de I'Éducation, 7(8), 113-128. Recuperado de http://socioconstructivismo.unizar.es/wp-content/uploads/2010/07/etnocientificidad.pdf

Simons, H. (2011). El estudio de caso: Teoría y práctica. Madrid: Morata.

Strauss, A. y Corbin, J. (1990). Basis of qualitative research. Grounded theory procedures and techniques. Newbury Park: Sage.

Vergara L., O., Hernández G., F. y Cárdenas R., R. (2009). Classroom research and professional development. Profile: Issues in Teachers' Professional Development, 11(2), 169-191.

Yin, R.K. (2009). Case study research: Design and methods. 4a. ed. Londres: Sage.

Zeichner, K.M. (2010). La formación del profesorado y la lucha por la justicia social. Madrid: Morata. 